\title{
A peculiar object in M 51: fuzzy star cluster or a background galaxy? ${ }^{\star}$
}

\author{
R. A. Scheepmaker, H. J. G. L. M. Lamers, S. S. Larsen, and P. Anders
}

\begin{abstract}
Astronomical Institute, Utrecht University, Princetonplein 5, 3584 CC Utrecht, The Netherlands
e-mail: scheepmaker@astro.uu.nl
\end{abstract}

Received 26 July 2007 / Accepted 19 October 2007

\section{ABSTRACT}

\begin{abstract}
Aims. We study a peculiar object with a projected position close to the nucleus of M 51. It is unusually large for a star cluster in M 51 and we therefore investigate the three most likely options to explain this object: (a) a background galaxy, (b) a cluster in the disk of M51 and (c) a cluster in M 51, but in front of the disk.

Methods. We use broad-band images of the Advanced Camera for Surveys and the Near Infrared Camera and Multi-Object Spectrometer, both on board the Hubble Space Telescope, to study the properties of this object. Assuming the object is a star cluster, we fit the metallicity, age, mass and extinction using simple stellar population models. Assuming the object is a background galaxy, we estimate the extinction from the colour of the background around the object. We study the structural parameters of the object by fitting the spatial profile with analytical models.

Results. We find de-reddened colours of the object which are bluer than expected for a typical elliptical galaxy, and the central surface brightness is brighter than the typical surface brightness of a disc galaxy. It is therefore not likely that the object is a background galaxy. Assuming the object is a star cluster in the disc of M 51, we estimate an age and mass of $0.7_{-0.1}^{+0.1} \mathrm{Gyr}$ and $2.2_{-0.3}^{+0.3} \times 10^{5} M_{\odot}$, respectively (with the extinction fixed to $E(B-V)=0.2$ ). Considering the large size of the object, we argue that in this scenario we observe the cluster just prior to final dissolution. If we fit for the extinction as a free parameter, a younger age is allowed and the object is not close to final dissolution. Alternatively, the object could be a star cluster in M 51, but in front of the disc, with an age of $1.4_{-0.2}^{+0.5} \mathrm{Gyr}$, mass $M=1.7_{-0.3}^{+0.8} \times 10^{5} M_{\odot}$. Its effective radius is between $\sim 12-25 \mathrm{pc}$. This makes the object a "fuzzy star cluster", raising the issue of how an object of this age would end up outside the disc.
\end{abstract}

Key words. galaxies: individual: M 51 - galaxies: star clusters

\section{Introduction}

Recently, Scheepmaker et al. (2007) studied the effective (halflight) radii of 1284 star clusters in the disc of M 51. The largest cluster candidate in their sample, carrying their ID number "212995", is remarkable in several ways.

If the object is a cluster in the disk of M 51, it is exceptionally large, with an estimated effective radius of $21.6 \mathrm{pc}$, and located at a galactocentric distance of $1 \mathrm{kpc}$ in a region with many dust lanes. A cluster with this size and galactocentric distance is not expected to survive for long in the spiral disc, where the disruptive effects of the tidal field and other external perturbations (spiral arms, GMCs) are large (Gieles et al. 2005; Gieles et al. 2006). If the cluster candidate is indeed in the disc, it should therefore be young and highly reddened to account for its observed colours of $B-V=0.75$ and $V-I=1.05$. Using only 2 colours, however, Scheepmaker et al. (2007) could not accurately determine an age and extinction.

Observations of HII regions in the disc of M 51 show that the metallicity of the gas is approximately solar to twice solar (Diaz et al. 1991; Hill et al. 1997). If the object is in the disc of M 51, we would therefore expect its metallicity to be around solar. Outside of the disc, the metallicity could be lower.

^ Based on observations made with the NASA/ESA Hubble Space Telescope, obtained from the data archive at the Space Telescope Institute. STScI is operated by the association of Universities for Research in Astronomy, Inc., under the NASA contract NAS 5-26555.
There is also the possibility that the object is a background galaxy. The observed colours of the object are similar to the colours of typical background galaxies, but this suggests that the reddening through the disc of M 51 is negligible. This would be quite remarkable at a distance of $1 \mathrm{kpc}$ from the nucleus, where the extinction is expected to be high, as suggested by the presence of numerous dust lanes. The hypothesis of a background galaxy therefore suggests a chance alignment, such that the object is observed through a "hole" in a region of the disc with many dust lanes.

In this work we present a more detailed study of the nature of the object by extending the data with archival HST/NICMOS observations in $F 110 \mathrm{~W}$ and $F 160 \mathrm{~W}$, and by estimating the extinction of the object. Having better constraints on the intrinsic spectral energy distribution of the object, we compare the two hypotheses, of the object being a star cluster or a background galaxy, in a quantitative way.

\section{Observations and photometry}

\subsection{Observations}

We make use of the HST/ACS dataset in the $F 435 \mathrm{~W}, F 555 \mathrm{~W}$ and $F 814 W$ bands of M 51 (NGC 5194). These data were taken as part of the Hubble Heritage Project in January 2005 under proposal ID 10452 (PI: S. V. W. Beckwith). We also used HST/NICMOS (NIC3) observations in the F110W and F160W bands from the archives (proposal ID 7237, PI: N. Scoville). The 


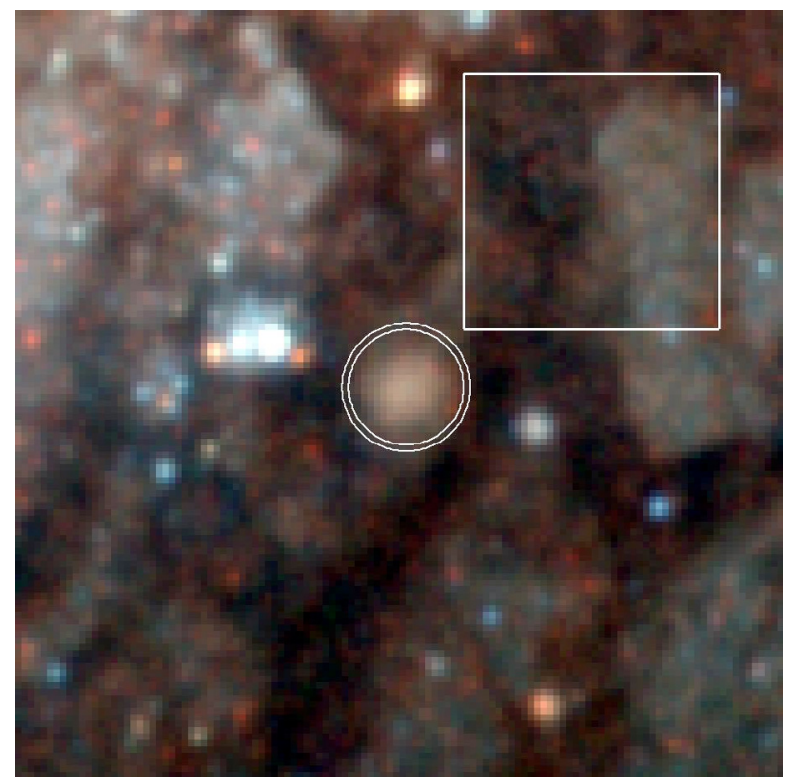

Fig. 1. Colour image of $120 \times 120$ pixels centred on the peculiar object " 212995 ". $F 435 W$ is blue, $F 555 W$ is green and $F 814 W$ is red. The two rings have a radius of 9 and 10 pixels, respectively. The box shows the $40 \times 40$ pixel region used for estimating the intrinsic colour of the background.

ACS dataset is described in more detail in Mutchler et al. (2005) and Scheepmaker et al. (2007). For the NICMOS dataset, we refer to Bastian et al. (2005).

Scheepmaker et al. (2007) located the peculiar object (carrying their ID number 212995$)$ at $\mathrm{RA}=13^{\mathrm{h}} 29^{\mathrm{m}} 51^{\mathrm{s}} .94$, Dec $=$ $+47^{\circ} 11^{\prime} 19^{\prime}$. 63 . A colour image of a $120 \times 120$ pixel region centred on the object is shown in Fig. 1, showing that the object is positioned in between two dark dust lanes.

Scheepmaker et al. (2007) used the ACS data to perform aperture photometry on the object. Their settings and aperture corrections were, however, optimised for star clusters with an effective radius of $\sim 3 \mathrm{pc}$. We discuss new aperture photometry on the object in Sect. 2.2, taking into account the larger size of the object and its position in between the dust lanes.

The effective radius of $21.6 \mathrm{pc}$ was measured by Scheepmaker et al. (2007), who used the ISHAPE routine (Larsen 1999, 2004) to convolve analytic surface brightness profiles with the point spread function (PSF). EFF (or Moffat) profiles with a power-law index of -1.5 were used (i.e. EFF15 profiles), because these were found to be the best-fitting profiles for young stellar clusters in the LMC (Elson et al. 1987). However, no tests were performed to find the best-fitting surface brightness profile for our specific object. We describe new fits to the surface brightness profile in Sect. 4, also using different analytic profiles.

\subsection{Photometry}

We performed aperture photometry on the object using the IRAF $^{1}$ /DAOPHOT package. For the ACS data an aperture with a radius of 8 pixels $\left(0.4^{\prime \prime}\right)$ was used, and for the background annulus we adopted an inner radius of 9 pixels and a width of 1 pixel. This annulus is indicated in Fig. 1. Zero points in the VEGAmag

1 The Image Reduction and Analysis Facility (IRAF) is distributed by the National Optical Astronomy Observatories, which are operated by the Association of Universities for Research in Astronomy, Inc., under cooperative agreement with the National Science Foundation.
Table 1. Results of the aperture photometry, corrected for Galactic foreground extinction.

\begin{tabular}{cc}
\hline \hline Filter & Magnitude (VEGAmag) \\
\hline$F 435 W(\sim B)$ & 22.13 \\
$F 555 W(\sim V)$ & 21.50 \\
$F 814 W(\sim I)$ & 20.49 \\
\hline$F 110 W(\sim J)$ & 20.08 \\
$F 160 W(\sim H)$ & 19.26 \\
\hline
\end{tabular}

system were taken from the ACS website. There is a possibility that the background annulus contains a small fraction of light from the object. However, this will leave the colours of the object, and therefore our results, largely unaffected, except for the value of the mass (Sect. 5.2), which will be a lower limit.

For the NICMOS data we used an aperture with a radius of 2 pixels $\left(0.4^{\prime \prime}\right)$ and a background annulus with an inner radius of 2 pixels and a width of 1 pixel. These values are consistent with the aperture of the ACS photometry. The magnitudes in the VEGAmag system were determined from the PHOTFNU values from the header, and the bandpass averaged flux densities for Vega from the on-line photometric keyword tables.

No aperture corrections were applied, since these are not well defined for an extended object of which the nature is not well known. However, the aperture was chosen to be as large as possible to minimise any systematic errors. Not applying any aperture correction will not change the colour of the objects significantly (assuming there are no colour gradients), since Scheepmaker et al. (2007) and Bastian et al. (2005) have shown that the aperture corrections are practically the same for the different filters we use.

To facilitate reading, we will sometimes refer to the HST filters using the extended Johnson-Cousin notation $(B V I J H)$, although we note that no transformations to this system have been applied. The results of the photometry are listed in Table 1.

\section{Extinction}

We have corrected the aperture photometry for Galactic foreground extinction according to Appendix B of Schlegel et al. (1998). Since the object is located in projection close to the nucleus of M $51\left(R_{\mathrm{G}}=1.02 \mathrm{kpc}\right)$, there is possibly a significant amount of additional extinction caused by the ISM of M 51. We used two methods to estimate this additional extinction of the object. We note that from here on, all quoted photometric values are already corrected for Galactic extinction, and any extinction we will mention refers to the additional extinction at the location of the object in M 51 .

\subsection{Extinction from cluster models}

The first method used to estimate the extinction assumes that the object is a star cluster, so we use cluster models to fit the extinction based on broad-band colours. We used the GALEV simple stellar population (SSP) models (Anders \& Fritze-v. Alvensleven 2003) together with the ANALYSED code (Anders et al. 2004) to simultaneously fit the extinction, metallicity, age and mass of the cluster, based on our broad-band photometry in the $F 435 \mathrm{~W}, \mathrm{F5} 55 \mathrm{~W}, \mathrm{~F} 814 \mathrm{~W}, \mathrm{~F} 110 \mathrm{~W}$ and $F 160 W$ bands. The Cardelli et al. (1989) standard extinction law was used $\left(R_{\mathrm{V}}=3.1\right)$. Table 4 shows the results for different initial constraints on the metallicity, which will be 
discussed in more detail in Sect. 5.2. These results are consistent with $E(B-V) \leq 0.7$, with a best-fitting $E(B-V)$ of 0.1 . The resulting ages and masses from these fits will be discussed in Sect. 5.2.

\subsection{Extinction from background colour}

The second method used to estimate the extinction of the object is based on comparing the average colour of a background annulus around the object to the intrinsic colour of the local background. The advantage of this method is that we do not have to assume the nature of the object (background galaxy or star cluster) a priori. However, the local background might not be smooth, but could show intrinsic colour variations. The intrinsic colour of the background is therefore not well defined and this method can therefore only provide a rough estimate of the extinction.

For the average observed colour of the background around the object we used the same annulus as we used for the aperture photometry (i.e. an inner radius of 9 pixels and a width of 1 pixel). After correcting for Galactic foreground extinction, the ring has a mean $B-V$ and $V-I$ of $0.75 \pm 0.06$ and $1.29 \pm 0.06$, respectively.

To estimate the intrinsic colour of the background we used the $40 \times 40$ pixel region indicated with the box in Fig. 1. This region was selected because it is free of point sources and close to the object, and contains a large range of pixel colours, which we assume to be mainly due to extinction differences (the region is at the border of a dust lane). We show the $B-V$ versus $V-I$ colour-colour diagram of all the pixels in this box in Fig. 2, which also shows the extinction vector. The observation that the scatter in this diagram is mostly parallel to the extinction vector is consistent with extinction being the dominant cause for colour variations among the pixels. Assuming that the pixels with the lowest values for $B-V$ and $V-I$ are least extincted, we estimate the upper limit for the intrinsic colour of the background to be $(B-V)_{0}=0.55 \pm 0.10$ and $(V-I)_{0}=1.00 \pm 0.10$.

We can estimate a lower limit for the extinction of the background ring around the object by assuming that the ring has the intrinsic colour of the background, and that the extinction is caused by a foreground sheet. Thus, the observed mean colours of the ring result in $E(B-V)=0.2 \pm 0.1$ and $E(V-I)=0.3 \pm 0.1$. It is more likely, however, that any dust present in the ring will be mixed with the stars. For the same sheet of dust, this reduces the amount of extinction estimated by the observed colours of the ring. We therefore conclude that if our object is in the disc, it has an extinction of at least $E(B-V)=0.2$. If the object is behind the disc, the extinction is likely to be even higher.

\section{Surface brightness profile}

In order to study the structural parameters of our object, we measured the surface brightness profile by performing aperture photometry in a series of circular rings around the object, measuring the average intensity in every ring as a function of radius. The resulting profile is shown in Fig. 3, in which we also plot the bestfitting surface brightness profiles as determined by the ISHAPE routine (Larsen 1999, 2004). IsHAPE fits two-dimensional analytic profiles to the surface brightness distribution of a source, taking into account the convolution with the PSF of the telescope. In Fig. 3 we show the best-fitting profiles typically used for fitting star clusters (i.e. a King 30 profile, see King 1962, and an EFF15 profile, see Elson et al. 1987) and the profile typically

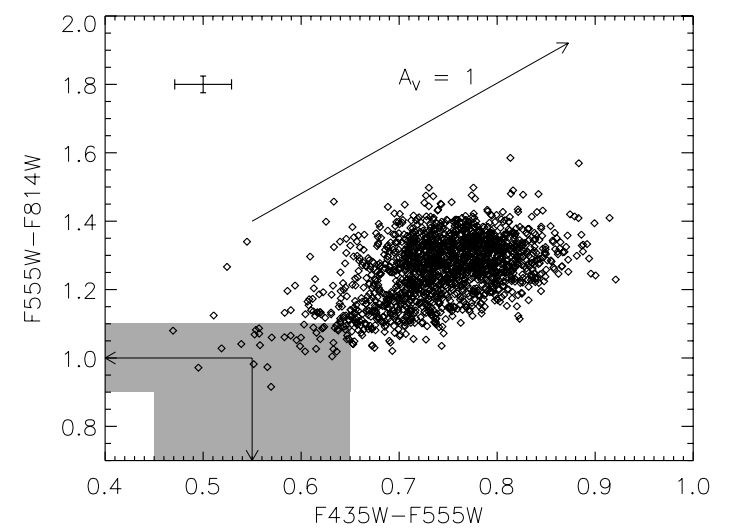

Fig. 2. $F 435 W-F 555 W$ versus $F 555 W-F 814 W$ diagram of the pixels in the background box, after correcting for Galactic foreground extinction. The extinction vector is the standard extinction law from Cardelli et al. (1989). The cross in the top-left corner shows the typical error of the pixel colours. The gray region is selected by eye and shows the uncertainty around the two values selected as the intrinsic colour of the background (indicated by the two arrows): $(B-V)_{0}=0.55 \pm 0.10$, $(V-I)_{0}=1.00 \pm 0.10$.

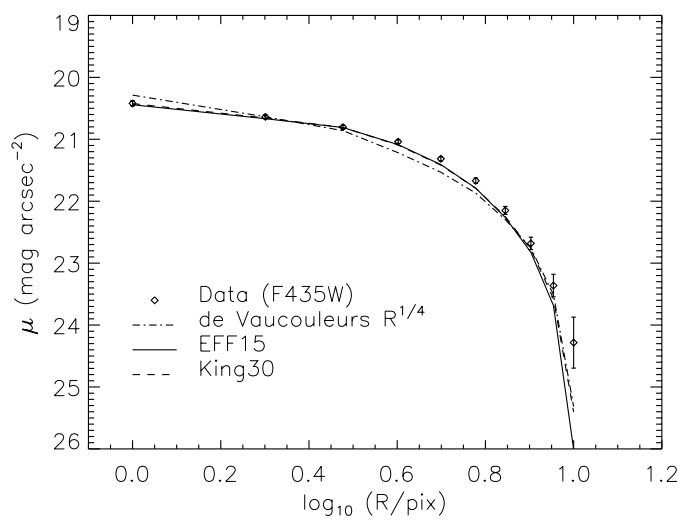

Fig. 3. Surface brightness profile of the object (including errorbars), compared to the best-fitting surface brightness profiles, assuming typical star cluster profiles (EFF15 and King 30) and the typical profile for elliptical galaxies (a de Vaucouleurs $R^{1 / 4}$ law).

used for fitting elliptical galaxies (a de Vaucouleurs $R^{1 / 4}$ profile, see de Vaucouleurs 1948). The surface brightness profiles of these best-fitting models were measured from the model images that ISHAPE creates. Therefore they include the convolution with the PSF and take into account the slight ellipticity of the object.

Assuming different analytic profiles, we find consistent results for the $F W H M$ and the aspect ratio $(a)$ of the object, namely $F W H M \approx 9$ pixels (along the major axis) and $a \approx 0.82$. Although the cluster profiles (King/EFF) provide better fits than a de Vaucouleurs profile, ISHAPE can not determine the bestfitting concentration/power-law index for these profiles, since the extended "wings" are affected by the dust lanes too much. The value for the best-fitting effective radius depends on this index, and therefore on the choice of the profile. In Table 2 we summarise the results of fitting the surface brightness distribution of our object, assuming different profiles. For the sake of completeness we also included the values for the de Vaucouleurs profile. Due to the much steeper decline in the innermost regions of a de Vaucouleurs profile, the FWHM of this profile is much smaller than the FWHM of the other profiles, and the effective radius is much larger. These values are not very meaningful, 
Table 2. Results of fitting different analytic profiles to the observed surface brightness distribution on the $F 435 W$ image, using the IsHAPE routine.

\begin{tabular}{lccc}
\hline \hline Profile & $F W H M($ pix $)$ & $R_{\text {eff }}($ pix $)$ & $R_{\text {eff }}(\mathrm{pc})^{a}$ \\
\hline de Vaucouleurs & 0.15 & $1.1 \times 10^{3}$ & - \\
EFF15 & 9.21 & 9.47 & $19.29^{b}$ \\
EFF25 & 9.34 & 5.78 & 11.77 \\
King 5 & 8.80 & 5.66 & 11.52 \\
King 30 & 9.07 & 12.15 & 24.74 \\
\hline
\end{tabular}

${ }^{a}$ Assuming a distance to M 51 of $8.4 \pm 0.6 \mathrm{Mpc}$ (Feldmeier et al. 1997); ${ }^{b}$ This value is slightly smaller than the $R_{\mathrm{eff}}=21.6 \mathrm{pc}$ measured by Scheepmaker et al. (2007), which is caused by the use of a larger "fitting radius" in the current study (i.e. 10 pixels).

Table 3. Intrinsic broad-band colours of the object, assuming different degrees of extinction and using the Galactic extinction law of Cardelli et al. (1989).

\begin{tabular}{cccc}
\hline \hline$E(B-V)$ & $(B-V)_{0}$ & $(V-I)_{0}$ & $(J-H)_{0}$ \\
\hline 0 & 0.63 & 1.00 & 0.82 \\
0.1 & 0.53 & 0.84 & 0.79 \\
$0.2^{a}$ & 0.43 & 0.68 & 0.76 \\
0.4 & 0.23 & 0.35 & 0.71 \\
0.6 & 0.03 & 0.03 & 0.65 \\
0.8 & -0.17 & -0.29 & 0.59 \\
1.0 & -0.37 & -0.62 & 0.54 \\
\hline
\end{tabular}

${ }^{a}$ This value is our best estimate for the average extinction in a ring around the object (Sect. 3.2).

however, since the de Vaucouleurs profile does not provide a good fit to the data.

For comparison, the effective radius of a typical star cluster is 2-3 pc (Scheepmaker et al. 2007; Jordán et al. 2005; Harris 1996), which, at the distance of M 51, is equivalent to a $F W H M \approx 1$ pixel. Thus, although the effective radius of the object is not well constrained without knowing the underlying profile, it is clear that if the object is at the distance of M 51, it is about 9-10 times larger than a typical star cluster.

\section{The nature of the object}

\subsection{Background galaxy}

If the object is a background galaxy, the mean extinction of the ring around the object (Sect. 3.2) is a lower limit for the mean extinction of the background galaxy. If we use an extinction of at least $E(B-V)=0.2$, as estimated in Sect. 3.2, to correct the observed colours, we find intrinsic colours of the background galaxy no redder than $(B-V)_{0}=0.43,(V-I)_{0}=0.68$ and $(J-H)_{0}=0.81$. In Table 3 we list the corrected colours for a range of extinctions.

We can compare the intrinsic colours of the background galaxy to the typical colours of other galaxies. Tinsley (1971) finds $(B-V)_{0} \approx 0.9$ for giant elliptical galaxies (after applying K-corrections), and using the catalog of Eisenhardt et al. (2007), we find a mean $(B-V)_{0}=1.15$ and $(V-I)_{0}=1.31$ for 486 galaxies in the Coma cluster (without K-corrections). Tonry et al. (2001) find a mean colour of $(V-I)_{0}=1.15 \pm 0.06$ for a survey of 300 (mostly E and S0) galaxies (without K-corrections). These colours are significantly redder than those estimated for our object. The colours used in these galaxy surveys are in the standard Johnson-Cousins system, and a direct comparison to the colours of the background galaxy could therefore be

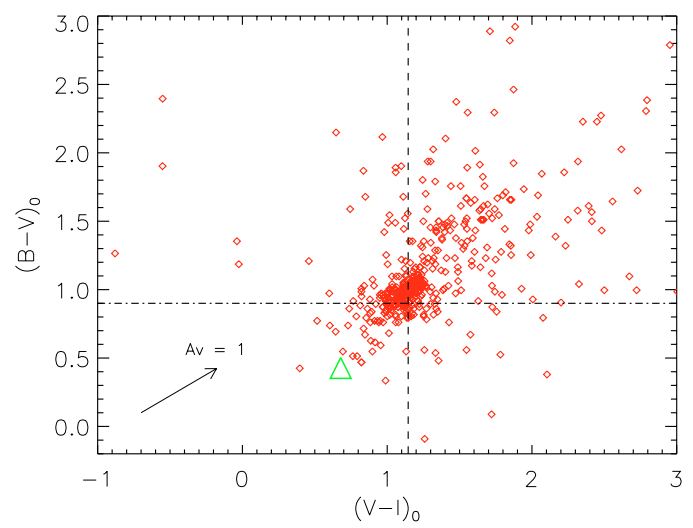

Fig. 4. $(B-V)_{0}$ versus $(V-I)_{0}$ diagram, showing the 486 galaxies from the Coma cluster (red diamonds), the mean $(B-V)_{0}$ of giant elliptical galaxies of Tinsley (1971) (horizontal line) and the mean $(V-I)_{0}$ of mostly E and S0 galaxies of Tonry et al. (2001) (vertical line). For comparison, we also show the estimated intrinsic colours of our object, assuming an extinction of $E(B-V)=0.2$ (green triangle).

misleading. However, Fukugita et al. (1995) give colours (not corrected for Galactic reddening) of galaxies with different morphological types in the $H S T / W F P C 2$ filter system. For E- and S0-type galaxies, these colours are $\left(V_{F 555 W}-I_{F 814 W}\right)=1.27$ and 1.12 , respectively. These colours are still significantly redder than those estimated for our object, and this suggests that the colour differences involved in the transformations between the photometric systems are small.

Applying K-corrections to the objects in the catalogue of Eisenhardt et al. (2007) or Tonry et al. (2001) would make their colours bluer, but also the colours of our object would have to be corrected for its K-correction, depending on its unknown distance. To reconcile the difference in colours between our object and these catalogue galaxies, the K-corrections of these galaxies have to be $\sim 0.6$ mag more negative (in $B-V$ and $V-I$ ) than those of our object. Considering the distance to the Coma cluster of $\sim 85 \pm 10 \mathrm{Mpc}$ (Jensen et al. 1999), the expected K-corrections are $\lesssim-0.1 \mathrm{mag}$ (Bicker et al. 2004). Therefore we conclude that $\mathrm{K}$-corrections cannot reduce the difference in colours between our object and the typical galaxies.

In Fig. 4 we compare the intrinsic colours of the object to the typical galaxy colours. If our object is a background galaxy, its $(B-V)_{0}$ and $(V-I)_{0}$ colours are significantly bluer than the mean $(B-V)_{0}$ and $(V-I)_{0}$ colours of elliptical galaxies, and also bluer than $98 \%$ and $97 \%$, respectively, of the galaxies of the Coma cluster.

Another comparison to elliptical galaxies can be made in terms of the surface brightness profile. The surface brightness profile of many bright elliptical galaxies is best described by a de Vaucouleurs $R^{1 / 4}$ law (de Vaucouleurs 1948). In Fig. 3 we compare the surface brightness profile of our object with a fit assuming a de Vaucouleurs law. The figure shows that a de Vaucouleurs law is steeper than the observed surface brightness profile (both an EFF15 and a King 30 profile for clusters provide a better fit to the data).

If our object is a background galaxy, it does not necessarily have to be an elliptical or S0 galaxy, and could be a disc galaxy. For disc galaxies, Freeman (1970) found a nearly constant central surface brightness of $\mu_{B}=21.65 \pm 0.30 \mathrm{mag} \mathrm{arcsec}{ }^{-2}$ for 28 out of 36 galaxies. 7 out of 36 galaxies had a surface brightness brighter than $\sim 20.1 \mathrm{mag} \operatorname{arcsec}^{-2}$. Barden et al. (2005) find $\mu_{B}=21.11 \pm 0.03 \mathrm{mag} \operatorname{arcsec}^{-2}$, increasing with redshift to $\mu_{B} \approx 19.7 \pm 0.07 \mathrm{mag} \operatorname{arcsec}^{-2}$ for $z=1$. Comparing these 
Table 4. Results of fitting different GALEV SSP models to the broad-band photometry of the object.

\begin{tabular}{clccccc}
\hline \hline$\#$ & Constraint & $Z / Z_{\odot}$ & Age $/ \mathrm{Gyr}$ & $M / 10^{5} M_{\odot}$ & $E(B-V)$ & Comment \\
\hline 1. & none & 0.4 & $1.2_{-1.2}^{+14.8}$ & $1.6_{-1.5}^{+7.5}$ & $0.1_{-0.1}^{+0.6}$ & \\
2. & $0.4 \leq Z / Z_{\odot} \leq 2.5$ & 0.4 & $1.2_{-1.2}^{+0.9}$ & $1.6_{-1.5}^{+1.2}$ & $0.1_{-0.1}^{+0.5}$ & \\
3. & $Z=Z_{\odot}$ & & $1.0_{-1.0}^{+0.4}$ & $1.9_{-1.8}^{+0.6}$ & $0.1_{-0.1}^{+0.4}$ & \\
4. & $Z / Z_{\odot}=0.4, E(B-V)=0$ & & $1.4_{-0.2}^{+0.5}$ & $1.7_{-0.3}^{+0.8}$ & & case B \\
5. & $Z=Z_{\odot}, E(B-V)=0.2$ & & $0.7_{-0.1}^{+0.1}$ & $2.2_{-0.3}^{+0.3}$ & & case A \\
6. & $Z=Z_{\odot}, E(B-V)=0.7$ & & $0.004^{\mathrm{a}}$ & $0.084^{\mathrm{a}}$ & & maximum extinction \\
\hline
\end{tabular}

${ }^{a}$ The errors in this best-fitting model can not be estimated due to the much lower probability of the remaining models.

values to our object, within a radius of 2 pixels our object has $\mu_{B} \leq 19.60 \mathrm{mag} \operatorname{arcsec}^{-2}$, corrected for Galactic foreground extinction, and an extinction of at least $E(B-V) \geq 0.2$ for the disc of M51. Our object therefore has a surface brightness which is brighter than the majority of the disc galaxies studied by Freeman (1970), but it is consistent with the brightest galaxies from this sample and with the highest redshift galaxies from Barden et al. (2005).

Assuming the lower-limit estimate of $E(B-V)=0.2$, we can therefore not exclude that the object is a background galaxy. We find, however, that for an elliptical galaxy, the object has $(B-V)_{0}$ and $(V-I)_{0}$ colours $\sim 0.6$ mag bluer than expected and a surface brightness profile that falls off less steeply than a $R^{1 / 4}$ law, while for a disc galaxy the surface brightness is remarkably high.

\subsection{Star cluster}

Assuming the object is a star cluster, it could either be located in the disc of M 51, or in front of the disc and seen in projection. For the latter case, the metallicity of the cluster could be different from the metallicity of the disc, and the extinction could be lower than the mean extinction of the ring around the object (Sect. 3.2). We used the GALEV SSP models described in Sect. 3.1 to fit the age, mass, extinction and metallicity of the cluster. We should note here, however, that not using $U$-band data in the SSP model fitting makes the derived ages and extinctions rather uncertain. Anders et al. (2004) have shown that not including $U$-band data can lead to underestimating the extinction and overestimating the ages of young clusters up to an order of magnitude. There are HST/WFPC2 $U$-band data of the region covering our object available in the archives. However, these data are not deep enough to detect our object. We cannot exclude the possibility that our object is younger than the best-fitting age determined from only using BVIJH. We will take this uncertainty into account when we draw our conclusions in Sect. 6.

The results of the fits, for different initial constraints, are summarised in Table 4. Without any initial constraints on the metallicity and extinction, the age and mass of the cluster are not well defined, although the best fit indicates $Z / Z_{\odot}=0.4$ and an extinction in the range $0-0.7$ in terms of $E(B-V)$. The lowest two metallicities $\left(0.02 Z_{\odot}\right.$ and $\left.0.2 Z_{\odot}\right)$ were fitted with a lower probability than a metallicity in the range $0.4 \leq Z / Z_{\odot} \leq 2.5$. We therefore exclude these lowest two metallicities, which reduces the maximum possible age and mass of the cluster to age $\leq 2.1 \mathrm{Gyr}$ and $M \leq 2.8 \times 10^{5} M_{\odot}$. A metallicity in the range $0.4 \leq Z / Z_{\odot} \leq 2.5$ is consistent with the observed metallicity in the inner disc of M 51 (Sect. 1).

If we fix the models to solar metallicity, we find an extinction of $E(B-V)=0.1_{-0.1}^{+0.4}$, which is roughly consistent with our independent estimate of the extinction in the disc from Sect. 3

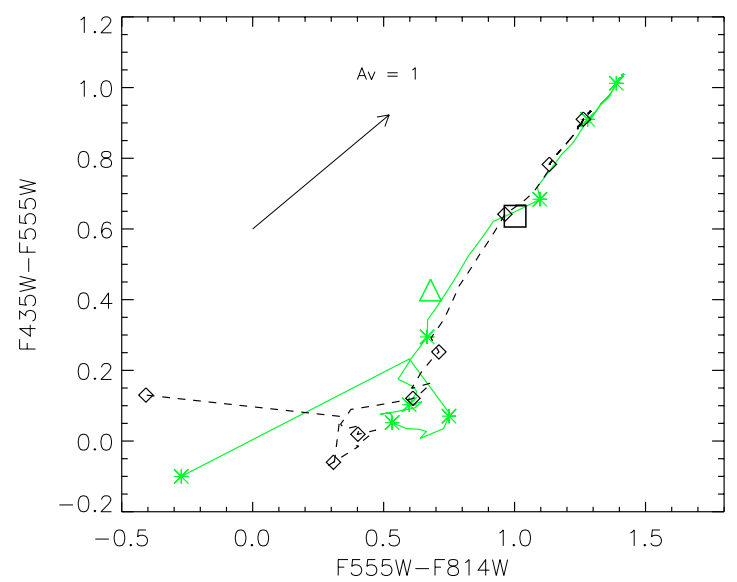

Fig. 5. $F 435 W-F 555 W$ versus $F 555 W-F 814 W$ diagram, showing the colour evolution with time of two GALEV SSP models (green/solid: $Z=0.02$, black/dashed: $Z=0.008$ ), and the object without extinction correction (black square) and with an extinction correction of $E(B-V)=0.2$ (green triangle). The symbols on the model curves indicate values of $\log (\mathrm{age})$, running from 6.6 (lower left) to 10.1 (top right) in steps of 0.5 dex.

(using the average background colour). We therefore consider two cases:

A. the cluster is located in the disc with an extinction of $E(B-V) \simeq 0.2$ and solar metallicity;

B. the cluster is seen in projection against the disc with an extinction of $E(B-V)=0.0$ and a metallicity of $Z / Z_{\odot}=0.4$.

In Fig. 5 we compare the two cases with the $F 435 W-F 555 W$ versus $F 555 W-F 814 W$ colour-colour evolution of GALEV SSP models for $Z / Z_{\odot}=0.4$ (black dashed curve) and $Z / Z_{\odot}=1$ (green solid curve).

Considering case A, we find a best-fitting age $=0.7_{-0.1}^{+0.1} \mathrm{Gyr}$ and $M=2.2_{-0.3}^{+0.3} \times 10^{5} M_{\odot}$. For case $\mathrm{B}$ we find an age of $1.4_{-0.2}^{+0.5} \mathrm{Gyr}$ and a mass of $M=1.7_{-0.3}^{+0.8} \times 10^{5} M_{\odot}$.

If the cluster is located in the disk of M 51 at a distance of only $1 \mathrm{kpc}$ from the centre (case A), one must question how it can have survived for such a long time. To this end we estimate its initial mass and the expected disruption time. For clusters losing mass due to stellar evolution and tidal dissolution, the present mass is related to the initial mass as

$M(t)=M_{i}\left\{\mu_{\mathrm{ev}}(t)^{\gamma}-\frac{\gamma t}{t_{0}} M_{i}^{-\gamma}\right\}^{1 / \gamma}$

with $M$ in $M_{\odot}$ (Lamers et al. 2005), where $\mu_{\mathrm{ev}}$ is the fraction of the initial mass that would have remained at age $t$, if stellar evolution had been the only mass loss mechanism. For $t=0.7 \mathrm{Gyr}$ and solar metallicity, one finds $\mu_{\mathrm{ev}}=0.77$. The parameters $t_{0}$ 
and $\gamma$ describe the mass loss due to dissolution of the cluster as $(\mathrm{d} M / \mathrm{d} t)_{\mathrm{dis}}=-M(t)^{1-\gamma} / t_{0}$ (Lamers et al. 2005). $N$-body simulations of clusters in tidal fields (Baumgardt \& Makino 2003) and $N$-body simulations of cluster dissolution due to shocks by encounters with giant molecular clouds or spiral arms (Gieles et al. 2006, 2007) both suggest that $\gamma \simeq 0.62$. This value agrees with the value that was derived empirically from cluster samples in different galaxies (Boutloukos \& Lamers 2003; Gieles et al. 2005). The value of the disruption parameter $t_{0}$ depends strongly on the local conditions. For clusters in M 51 at $1<R<5 \mathrm{kpc}$, Gieles et al. (2005) find a mean value of $t_{0}=6.6 \times 10^{5} \mathrm{yr}$. Adopting these values, Eq. (1) implies an initial mass of $M_{i} \simeq 4.5 \times 10^{5} M_{\odot}$, and we find that the cluster has lost about $50 \%$ of its initial mass.

In this estimate we have neglected the dependence of the dissolution time with galactocentric distance and the large radius of the cluster. The dissolution time of clusters is expected to decrease with galactocentric distance as $t_{0} \propto R_{G} / V$, where $V$ is the circular velocity at distance $R_{G}$ from the centre (Baumgardt \& Makino 2003; Gieles et al. 2005). This implies that $t_{0}$ could be smaller than the value adopted above by as much as a factor two or three. In that case, Eq. (1) implies an initial mass of about $6-9 \times 10^{5} M_{\odot}$, and the cluster has lost about $70 \%$ of its mass.

We note that the cluster, having a $R_{\text {eff }}$ of $\sim 12-25$ pc (depending on the assumed brightness profile, see Table 2), is very extended and "fuzzy". The tidal radius of a $M=2.2 \times 10^{5} M_{\odot}$ cluster at a galactocentric distance of $1.02 \mathrm{kpc}$ is

$r_{t}=\left(\frac{G M}{2 V_{\mathrm{G}}^{2}}\right)^{1 / 3} R_{\mathrm{G}}^{2 / 3} \approx 23.9 \mathrm{pc}$,

where we used $V_{\mathrm{G}}=200 \mathrm{~km} \mathrm{~s}^{-1}$ (García-Burillo et al. 1993). Therefore, if the object is a cluster in the disc of M 51, its effective radius of $\sim 12-25 \mathrm{pc}$ is very close to the tidal radius (within a factor $\sim 0.5-1$ ). This is remarkable, since such an extended cluster would not be able to stay bound at this location in the disc.

The disruption time of a cluster depends implicitly on the radius through the parameter $t_{0}$. For disruption in a tidal field, $t_{0} \propto$ $t_{\mathrm{rh}} \propto M^{1 / 2} r_{\mathrm{h}}^{3 / 2}$ (Spitzer 1987), in which $t_{\mathrm{rh}}$ is the half-mass relaxation time and $r_{\mathrm{h}}$ is the half-mass radius. This suggests that more extended clusters have a longer disruption time compared to compact clusters. However, for the disruption timescale $\left(t_{\text {dis }}\right)$ of clusters due to external perturbations (e.g. shocks due to encounters with GMCs) Spitzer (1958) has derived that $t_{\text {dis }} \propto M r_{\mathrm{h}}^{-3}$, implying that a large cluster is more susceptible to external disruptive effects (see also Gieles et al. 2006). Considering the location of the cluster close to the centre of M 51 in a region with many dust lanes, the external perturbations are likely to dominate in the disruption, and they will shorten the disruption time for such an extended cluster, compared to the disruption time of a cluster with a typical size of about $3 \mathrm{pc}$. Therefore, the estimated value of the disruption time parameter $t_{0} \sim 2-3 \times 10^{5} \mathrm{yr}$ would be too large. Using Eq. (1), this implies that the initial mass of such an extended cluster is probably much higher, to explain its current mass and age. This could mean that the cluster has lost significantly more than $70 \%$ of its initial mass, and is currently uncomfortably close to final dissolution. Although such a scenario is not impossible, it is unlikely that we observe a cluster in this last phase of its lifetime.

Case B, seeing the cluster in projection against the disc, is considered to be more likely. In this scenario the cluster can be located well outside the disc, where the tidal radius will be larger and the expected disruption time will be longer. This means that the initial mass needs to be lower compared to case A, and that we observe the cluster in a more "calm" phase of its lifetime.

To establish the nature of the object with greater certainty than presented in this study requires spectroscopic observations. These will be challenging, however, considering the low brightness of the object (Table 1). Deep $U$-band imaging, obtained in good seeing conditions, would also help establish the age and extinction with greater certainty.

\section{Conclusions}

We have studied the photometry, extinction and structural parameters of a peculiar object in M51 using HST/ACS and HST/NICMOS data. We have considered different possible scenarios for the object, from which we conclude the following:

1. If we assume that the object is a background galaxy, we estimate that the extinction through the disc of M 51 is at least $E(B-V)=0.2$. Correcting for this minimum extinction, we find intrinsic colours of $(B-V)_{0}=0.43,(V-I)_{0}=0.68$ and $(J-H)_{0}=0.76$. These colours suggest that the object is not a background galaxy, because $(B-V)_{0}$ and $(V-I)_{0}$ are $\sim 0.6$ mag bluer than expected for a typical elliptical galaxy (Fig. 4) and the surface brightness is $\sim 2 \mathrm{mag} \mathrm{arcsec}^{-2}$ brighter than a typical disc galaxy. If the extinction would be larger than $E(B-V)=0.2$, these differences in colour and surface brightness would be even larger.

2. The surface brightness distribution can be well approximated by typical cluster profiles (i.e. EFF or King profiles) with a $F W H M$ of $\sim 9$ pixels. A de Vaucouleurs $R^{1 / 4}$ law, typical for elliptical galaxies, does not provide a good fit to the inner part of the observed profile (Fig. 3). Because the exact concentration (or power-law) index of the object can not be determined, the effective radius of the object depends on the choice of the profile. Assuming the object is at the distance of M 51, our best estimates for the effective radius are between $\sim 12 \mathrm{pc}$ and $\sim 25 \mathrm{pc}$ (Table 2).

3. If we assume that the object is a star cluster in the disc of M51, we estimate an age of $0.7_{-0.1}^{+0.1} \mathrm{Gyr}$ and a mass $M=2.2_{-0.3}^{+0.3} \times 10^{5} M_{\odot}$, assuming $E(B-V)=0.2$ and solar metallicity. Considering the large size of the object, however, we estimate that in this scenario the cluster must initially have been very massive $\left(>4.5 \times 10^{5} M_{\odot}\right)$, and that we observe the cluster just prior to final dissolution. Although not impossible, this scenario is considered to be unlikely.

4. We can not fully exclude the possibility that our best-fitting cluster ages and extinctions are over- and underestimated, respectively, due to the lack of $U$-band photometry of our object. If we consider the maximum-fitted extinction of $E(B-V)=0.7$ (model 6 in Table 4), instead of the bestfitted extinction, the cluster has an age of only $\sim 4 \mathrm{Myr}$ and is not close to final dissolution. With our current data, however, the likelihood of this fit is very low.

5. If we assume that the object is a star cluster well outside the disc of M 51 and seen in projection against it, we estimate an age of $1.4_{-0.2}^{+0.5} \mathrm{Gyr}$ and a mass of $M=1.7_{-0.3}^{+0.8} \times 10^{5} M_{\odot}$. This scenario naturally explains why the best-fitting extinction is low, $E(B-V)=0.1_{-0.1}^{+0.5}$, although we observe the cluster in between two dust lanes. Furthermore, this scenario implies that we are not observing the cluster in a special phase of its lifetime.

6. We conclude that, without additional $U$-band data or spectroscopy, both the "disc" and "projection" scenarios are possible within the uncertainties. With the current data, however, 
the cluster models favour the scenario in which the object is a star cluster seen in projection against the disc. In this scenario, the cluster just qualifies as being a "faint fuzzy" star cluster, namely having $(B-V)_{0}>0.6,(V-I)_{0}>1.0$ and $R_{\text {eff }}>7$ pc (Larsen \& Brodie 2000). Seen in projection against the spiral disc, the (projected) galactocentric distance of $1 \mathrm{kpc}$ is not unexpected. However, with an estimated age of $1.4_{-0.2}^{+0.5} \mathrm{Gyr}$, the formation mechanism of such a cluster, in combination with its extended shape and its current location outside of the spiral disc, are interesting issues that leave room for further investigation.

Note added in proof: After the paper was accepted we became aware of the study of background galaxies seen though the disk of M51 by Holwerda et al. (2005). These authors found that the average opacity values for M 51 are the highest for any disk $\left(A_{I}=1.5\right)$, making it very unlikely that the object is a background galaxy.

Acknowledgements. We thank the International Space Science Institute (ISSI) in Bern, Switzerland, where part of this research took place. The anonymous referee is thanked for useful comments and suggestions.

\section{References}

Anders, P., \& Fritze-v. Alvensleven, U. 2003, A\&A, 401, 1063

Anders, P., Bissantz, N., Fritze-v. Alvensleben, U., \& de Grijs, R. 2004, MNRAS, 347, 196

Barden, M., Rix, H.-W., Somerville, R. S., et al. 2005, ApJ, 635, 959

Bastian, N., Gieles, M., Lamers, H. J. G. L. M., Scheepmaker, R. A., \& de Grijs, R. 2005, A\&A, 431, 905

Baumgardt, H., \& Makino, J. 2003, MNRAS, 340, 227
Bicker, J., Fritze-v. Alvensleben, U., Möller, C. S., \& Fricke, K. J. 2004, A\&A, 413, 37

Boutloukos, S. G., \& Lamers, H. J. G. L. M. 2003, MNRAS, 338, 717

Cardelli, J. A., Clayton, G. C., \& Mathis, J. S. 1989, ApJ, 345, 245

de Vaucouleurs, G. 1948, Ann. Astrophys., 11, 247

Diaz, A. I., Terlevich, E., Vilchez, J. M., Pagel, B. E. J., \& Edmunds, M. G. 1991, MNRAS, 253, 245

Eisenhardt, P. R., De Propris, R., Gonzalez, A. H., et al. 2007, ApJS, 169, 225

Elson, R. A. W., Fall, S. M., \& Freeman, K. C. 1987, ApJ, 323, 54

Feldmeier, J. J., Ciardullo, R., \& Jacoby, G. H. 1997, ApJ, 479, 231

Freeman, K. C. 1970, ApJ, 160, 811

Fukugita, M., Shimasaku, K., \& Ichikawa, T. 1995, PASP, 107, 945

García-Burillo, S., Combes, F., \& Gerin, M. 1993, A\&A, 274, 148

Gieles, M., Bastian, N., Lamers, H. J. G. L. M., \& Mout, J. N. 2005, A\&A, 441, 949

Gieles, M., Portegies Zwart, S. F., Baumgardt, H., et al. 2006, MNRAS, 371, 793 Gieles, M., Athanassoula, E., \& Portegies Zwart, S. F. 2007, MNRAS, 376, 809 Harris, W. E. 1996, AJ, 112, 1487

Hill, J. K., Waller, W. H., Cornett, R. H., et al. 1997, ApJ, 477, 673

Holwerda, B. W., Gonzalez, R. A., Allen, R. J., \& van der Kruit, P. C. 2005, AJ, 129,1396

Jensen, J. B., Tonry, J. L., \& Luppino, G. A. 1999, ApJ, 510, 71

Jordán, A., Côté, P., Blakeslee, J. P., et al. 2005, ApJ, 634, 1002

King, I. 1962, AJ, 67, 471

Lamers, H. J. G. L. M., Gieles, M., Bastian, N., et al. 2005, A\&A, 441, 117

Larsen, S. S. 1999, A\&AS, 139, 393

Larsen, S. S. 2004, A\&A, 416, 537

Larsen, S. S., \& Brodie, J. P. 2000, AJ, 120, 2938

Mutchler, M., Beckwith, S. V. W., Bond, H. E., et al. 2005, BAAS, 37

Scheepmaker, R. A., Haas, M. R., Gieles, M., et al. 2007, A\&A, 469, 925

Schlegel, D. J., Finkbeiner, D. P., \& Davis, M. 1998, ApJ, 500, 525

Spitzer, L. 1958, ApJ, 127, 17

Spitzer, L. 1987, Dynamical evolution of globular clusters, Princeton Series in Astrophysics (Princeton University Press)

Tinsley, B. M. 1971, Ap\&SS, 12, 394

Tonry, J. L., Dressler, A., Blakeslee, J. P., et al. 2001, ApJ, 546, 681 